Combining temperature and body-mass dependence ${ }^{1}$ is an interesting attempt to find out how the biological time clock works, but the clock seems to be imprecise. Data are presented on the development time for marine fish eggs in Fig. 2 of Gillooly et al. ${ }^{1}$, who infer that the intercept is similar to that in their Fig. 1, but when these are plotted on the same $y$-axis scale they are strikingly different. Also, egg-development times for univoltine aquatic insects ${ }^{9}$ were excluded from their compilation. Taking an arbitrary temperature of $20^{\circ} \mathrm{C}$, the biological clock runs fast by 20 days for univoltine aquatic insects and slow by 5 days for marine fish (Fig. 1c). The intercepts are
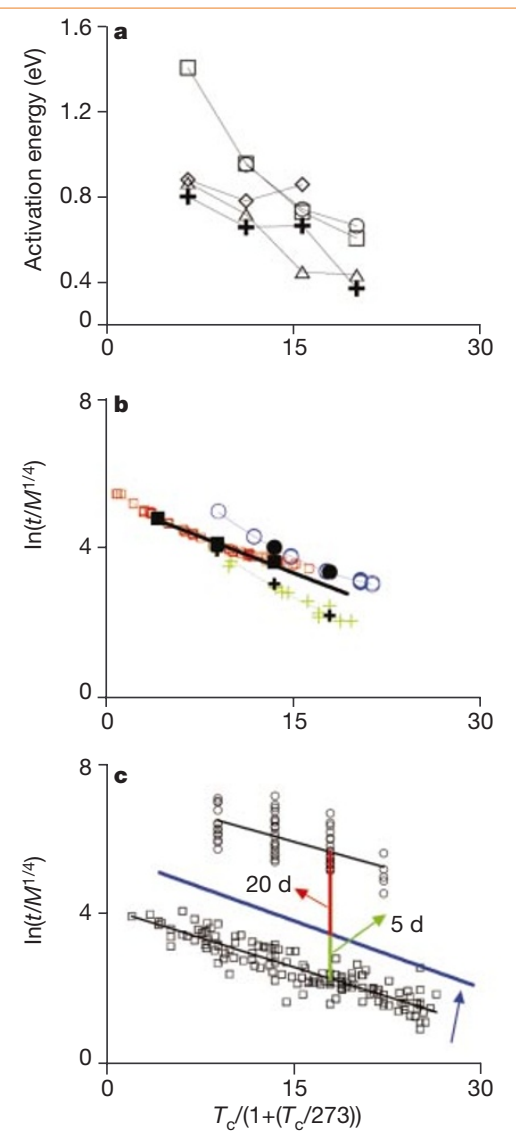

Figure 1 Effect of temperature on mass-corrected development time. a, Activation energy, $\bar{E}$, calculated following Gillooly et al. ${ }^{1}$, for different temperature intervals plotted against the interval's mean temperature. Lines link the average activation energy for species of amphibians (squares), fish (rhomboids), multivoltine aquatic insects (circles) and zooplankton (crosses, embryonic development; triangles, post-embryonic development). b, Colour symbols show the complete data sets, not adjusted to $5{ }^{\circ} \mathrm{C}$ intervals, for chinook salmon (squares), garfish (circles) and bream (crosses). Data sources from references in Gillooly et al. ${ }^{1}$. Black symbols are the adjusted data for each of these species as used by Gillooly et al. ${ }^{1}$; black line is their linear fit for fish (their Fig. 1b). c, 'Biological time clock' line (blue arrow) obtained by Gillooly et al. ${ }^{1}$ for aquatic ectotherms and birds (their Fig. 3). Red and green lines show prediction error of the 'biological time clock' at $20^{\circ} \mathrm{C}$ for non-diapause eggs of univoltine aquatic insects (circles) and marine fish (squares) incubated at different constant temperatures. Errors in days were calculated from egg masses of 0.01 and $0.9 \mathrm{mg}$, the average for each group from Gillooly et al. ${ }^{1}$. therefore not similar, cautioning against the conclusion that the model of Gillooly et al. is common and invariant to all organisms.

\section{Ángel López-Urrutia}

Plymouth Marine Laboratory, The Hoe,

Plymouth PL1 3DH, UK

e-mail:alop@pml.ac.uk

1. Gillooly, J. F., Charnov, E. L., West, G. B., Savage, V. M. \&

Brown, J. H. Nature 417, 70-73 (2002).

2. Gillooly, J. F. et al. Science 293, 2248-2251 (2001).

3. Ludwig, D. Physiol. Zool. 1, 358-389 (1928).

4. Bělehrádek, J. Protoplasma Monographien 8 (1935).

5. Crozier, W. J. Proc. Natl Acad. Sci. USA 10, 461-464 (1924).

6. Bělehrádek, J. Nature 118, 117-118 (1926).

7. Heilbrunn, L. V. Science 62, 268-269 (1925).

8. Bělehrádek, J. Nature 118, 478-480 (1926).

9. Gillooly, J. F. \& Dodson, S. I. Freshwat. Biol. 44, 595-604 (2000).

Gillooly et al. reply - Rombough asserts that our model ${ }^{1}$ is of limited use in predicting the temperature dependence of developmental time, but he has used a model with the same two primary variables, size and temperature, for the same purpose ${ }^{2}$. From his Fig. 1d above, he says that diversity among species is the salient feature for temperature dependence.

We replotted these data as the natural logarithm of mass-corrected incubation time against average incubation temperature in ${ }^{\circ} \mathrm{C}$ for fish with sizes and temperatures reported $\left(y=-0.18 x+4.34 ; \quad r^{2}=0.72 ; \quad n=36 ; \quad Q_{10}\right.$ ranges from 2 to 7 ; see supplementary information). The slope $(-0.18)$ is close to the value predicted by our model $(-0.14)$. Our model explains $72 \%$ of the variation, leaving only $28 \%$ to be explained by species differences, measurement errors and all other factors.

Rombough uses data on time intervals between stages to argue that the effect of temperature varies significantly during development. Most of this variation is measurement error because stages are defined arbitrarily, and times between them are short. Plotting cumulative times for three different stages from these data (Fig. 1) gives excellent fits and very similar slopes, showing that the temperature dependence remains nearly constant throughout development. Rombough actually presents additional support for our model.

López-Urrutia’s main theoretical objection is that "vital action is arrested in the vicinity of $0{ }^{\circ} \mathrm{C}$ ", so our model is undefined. Biological activity ceases at around $0{ }^{\circ} \mathrm{C}$ because of a phase transition, the freezing of water. We consider this to be a separate process from molecular kinetics (which ceases at absolute zero). Therefore, by extrapolating the $a(T)$ curve for $T>0$ to $T=0$, we obtain a $y$-intercept, $a(0)$, that is always non-zero.López-Urrutia argues that activation energy $(E)$ decreases systematically with temperature, and our figures only seem to be linear because we have averaged across temperatures for species. Plotting $E$ for narrow temperature intervals, as in his Fig. 1a, is subject to measurement errors; the confidence intervals are infinite. Systematic changes in $E$ with $T$ should give curvilinear relationships in plots of $\ln \left(t / m^{1 / 4}\right)$, as in his Fig. $1 b, c$ or our Fig. 1. Although one species seems

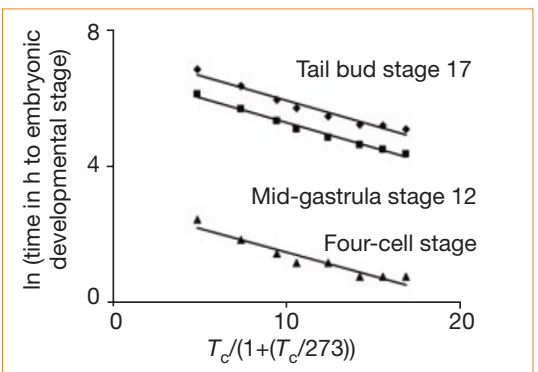

Figure 1 Plot of the natural logarithm of time to reach a specific embryonic development stage against incubation temperature for the tailed frog in Rombough's Fig. 1b (ref. 2). The slope of each line is close to the value predicted by our model $(-0.13)$. For the tail-bud stage 17, $y=-0.15 x+7.4$ and $r^{2}=0.96$; for midgastrula stage 12, $y=-0.15 x+6.7$ and $r^{2}=0.98$; and for the four-cell stage, $y=-0.14 x+2.8$ and $r^{2}=0.92$.

slightly curvilinear in his Fig. 1b, most species showed linear relationships across all our data.

Statistics provide a definitive answer for linearity. Curvilinear regression models (polynomials) did not give significantly better fits for any of our plots. Linearity is evident in López-Urrutia's Fig. 1c and in our Fig. 1, where linear fits account for $92-98 \%$ of the variation. Note that there is no averaging across temperatures for the fish in his Fig. 1c because each point represents a different species; all points in our Fig. 1 are for the same species.

In his Fig. 1c, López-Urrutia uses differences in intercepts, $a$, between fish in the wild, univoltine insects and the average for several taxonomic groups to question the reliability of the biological time clock. In our model, the coefficient $a$ allows for variation in intercepts with metabolic rate, $B_{0}$, and hence for differences in development times depending on which taxa, environmental conditions and developmental stage are measured. It is the $M^{-1 / 4}$ dependence on body size and $e^{-E / k T}$ temperature dependence in our equation (6), not the coefficient $a$, that defines the biological time clock. Note, however, that in LópezUrrutia's Fig. 1c, the slopes and therefore the $E$ values are nearly identical.

Both authors overlook our central message by focusing on temperature. We never claimed to have derived the Boltzmann factor or $Q_{10}$. Our model does provide a theoretical framework that combines the effects of size, temperature and stoichiometry to explain most of the variation in developmental rates across diverse environments and taxonomic groups.

James F. Gillooly ${ }^{\star}$, Eric L. Charnov ${ }^{\star}$, James H. Brown $^{\star}$, Van M. Savage $\dagger$, Geoffrey B. West $\dagger$ ${ }^{\star}$ Biology Department, University of New Mexico, Albuquerque, New Mexico 87131, USA

e-mail:gillooly@unm.edu

$\dagger$ Theoretical Division, Los Alamos National Laboratory, Los Alamos, New Mexico 87545, USA

1. Gillooly, J. F., Charnov, E. L., West, G. B., Savage, V. M. \& Brown, J. H. Nature 417, 70-73 (2002).

2. Rombough, P. J. in Global Warming: Implications for Freshwater and Marine Fish (eds Wood, C. M. \& McDonald, D. G.) 177-223 (Cambridge Univ. Press, 1997).

Supplementary information accompanies this communication on Nature's website. 